
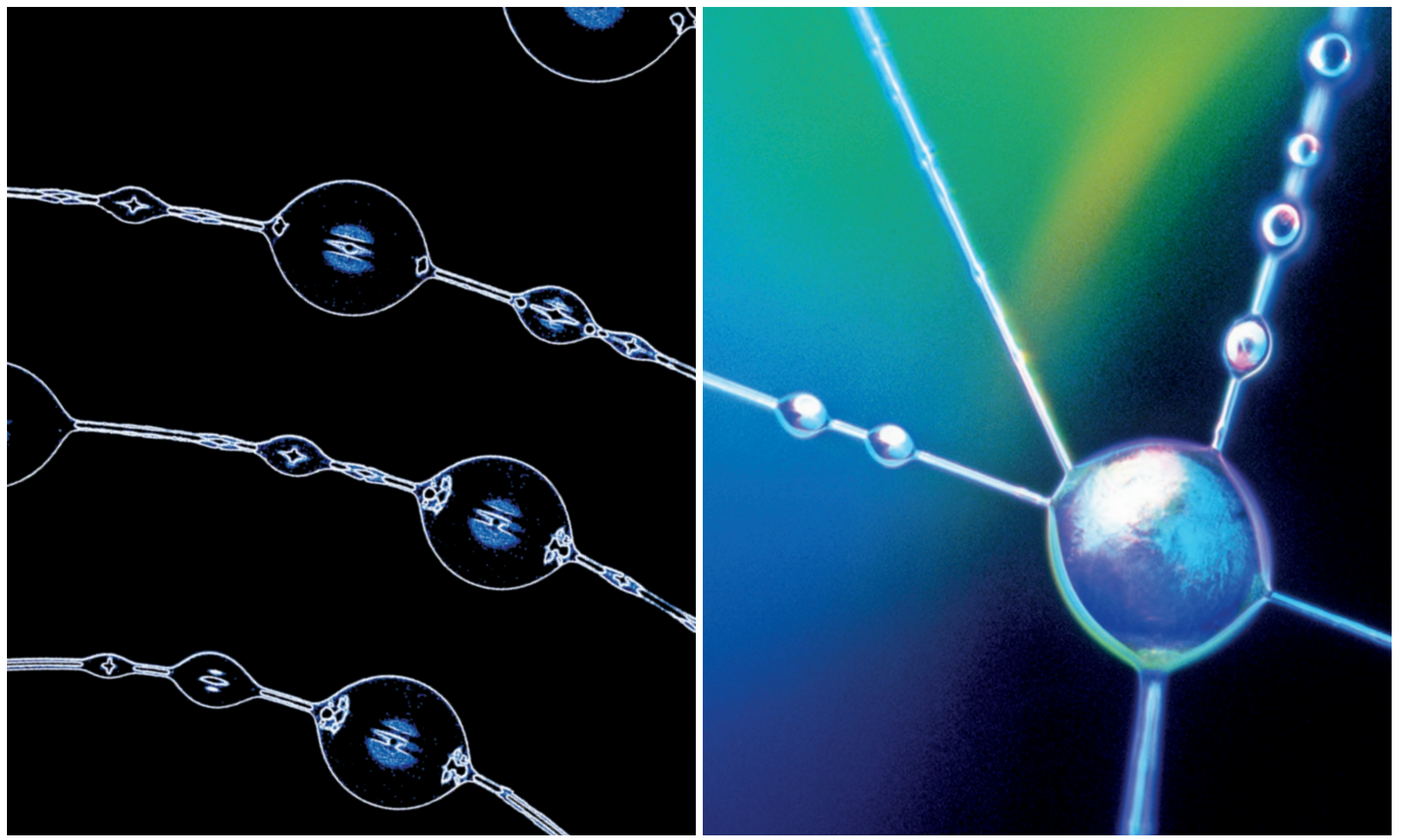

A synthetic mimic (left) of natural spider silk (right) incorporates polymer drops that can be used as a drug-delivery system.

SPIDERS

\title{
Web of intrigue
}

\section{The sturdy, stretchy, sticky silks spun by spiders have inspired engineers to design pioneering medical devices such as artificial tendons and corneas.}

\section{BY KATHERINE BOURZAC}

A Madagascan bark spider releases a silk dragline into the air. The wind carries the thin threads to the other side of a river, where they land on foliage on the opposite bank 25 metres away. The spider (Caerostris darwini) then stretches the bridgeline to establish tension, reinforces it, and draws on a palette of other silks, stretchier or stickier as needed, to fashion a web to capture the bugs flying over the water ${ }^{1}$.

C. darwini's bridging silk is the world's toughest known biomaterial - it is even tougher than steel (see 'The toughest thread'). But $C$. darwini's versatility in producing different kinds of silk is not unique. Many spiders can spin several silks: stiff, structural strands to stabilize their webs; gooey, stretchy spirals to capture flying insects; adhesive pads to anchor their homes in place; and extraordinarily robust draglines from which to hang.

The remarkable mechanical properties of these natural fibres have attracted the attention of materials scientists. Researchers are looking to arachnids and other silk makers for ideas about how to make new structural materials for bridges and vehicles, dirt-resistant adhesives for climbing robots and sturdy polymers for biomedical devices. Many silks bring together properties that are not readily present in manmade materials - the extreme toughness and elasticity seen in spider threads is one example. Silk proteins can be moulded like plastic or perform optical functions like silicon. Yet because they're organic, biological materials, silks are environmentally friendly and biocompatible. Silk proteins can be fashioned into films that can be implanted in the body, releasing drugs as they dissolve. This combination of features is unavailable in polyester or collagen or anything else, says David Kaplan, an early proponent of high-tech biomedical silk at Tufts University in

\section{$\rightarrow$ NATURE.COM}

Read about spider genomes at:

go.nature.com/jwvsc4
Medford, Massachusetts. "There's clearly a need for new biomaterials," he says. For Kaplan and others, silk is the best way to meet that need.

\section{COMMON THREAD}

Silk evolved independently in many invertebrates, including spiders, honeybees and silkworms. Individual spiders can make as many as six different kinds of silk proteins (and two glue proteins), each of which has evolved over the creatures' 400 million years of natural history. Each spider species uses its own variations of these proteins to make many different types of thread.

"We think that a primordial spider had one kind of silk, and then there were multiple events when the gene duplicated and evolved," says Cheryl Hayashi, a spider specialist at the University of California, Riverside. The species that are more closely related to these ancestors, such as tarantulas and trapdoor spiders, make silks of simple designs - messy tangles to trap walking insects, for example, using fewer types 
of silk. Other spiders evolved to make more complex spiralling orb webs, in which different regions are composed of different kinds of silk — some optimized for capturing prey, others for structural support of large web designs.

This evolutionary bounty has happy implications for engineers looking to put spider silk into human service. If a design calls for a fibre with a particular ratio of strength to stretchiness, "it's probably already been invented" by one of the tens of thousands of types of spider, says Hayashi.

Most research has centred on taking advantage of the toughness of spider silk - in materials science, toughness is a measure of how much energy it takes to break something. Materials such as spider silk are both strong and elastic. A large insect that flies into a spiderweb at top speed stretches the superfine fibres in the web but does not break them.

The toughest silks are found in spider draglines, which researchers are studying intensely. Spiders use draglines to dangle safely, to make the frames of their webs, and for situations in which resistance to breakage is paramount. In a scene from the 2004 movie Spider-Man 2, the eponymous superhero stops a runaway New York City subway train with his webbing, which is not too far of a stretch from reality. In 2012, physics graduate students at the University of Leicester, UK, calculated that dragline silk spun by the Darwin's bark spider would be able to perform a feat just as shown in the film.

If it could be manufactured, the Darwin's bark spider's dragline silk would be valuable for use in applications in which a lightweight, yet very strong, material is crucial: helmets for fighter pilots, artificial tendons and ligaments, or Spider-Man-like climbing ropes. But spiders are, from a practical, manufacturing point of view, useless. Spiders become cannibals when kept in captivity, and so must be caught from the wild. To obtain natural spider silk, a researcher must anaesthetize a spider, attach the end of one of its fibres to a variable-speed motor from an electric drill, then gently pull the thread out of one of the spider's glands. Depending on the species of spider, this technique can yield up to 100 metres of silk. This painstaking process is easier than picking apart the threads from spiderwebs, but it is not scalable. So researchers have been working on how to generate spider silks without spiders. The process presents two initial challenges: first, where to find the proteins; and second, how to assemble the proteins into a useful fibre.

\section{FIBRE FOCUS}

To realize the dream of artificial spider silk as an engineering material, researchers have started with genes. Randolph Lewis, a bioengineer at Utah State University in Logan, sequenced $^{2}$ the first spider-silk protein gene in 1990. Lewis chose the dragline of Nephila clavipes, a species indigenous to the Americas

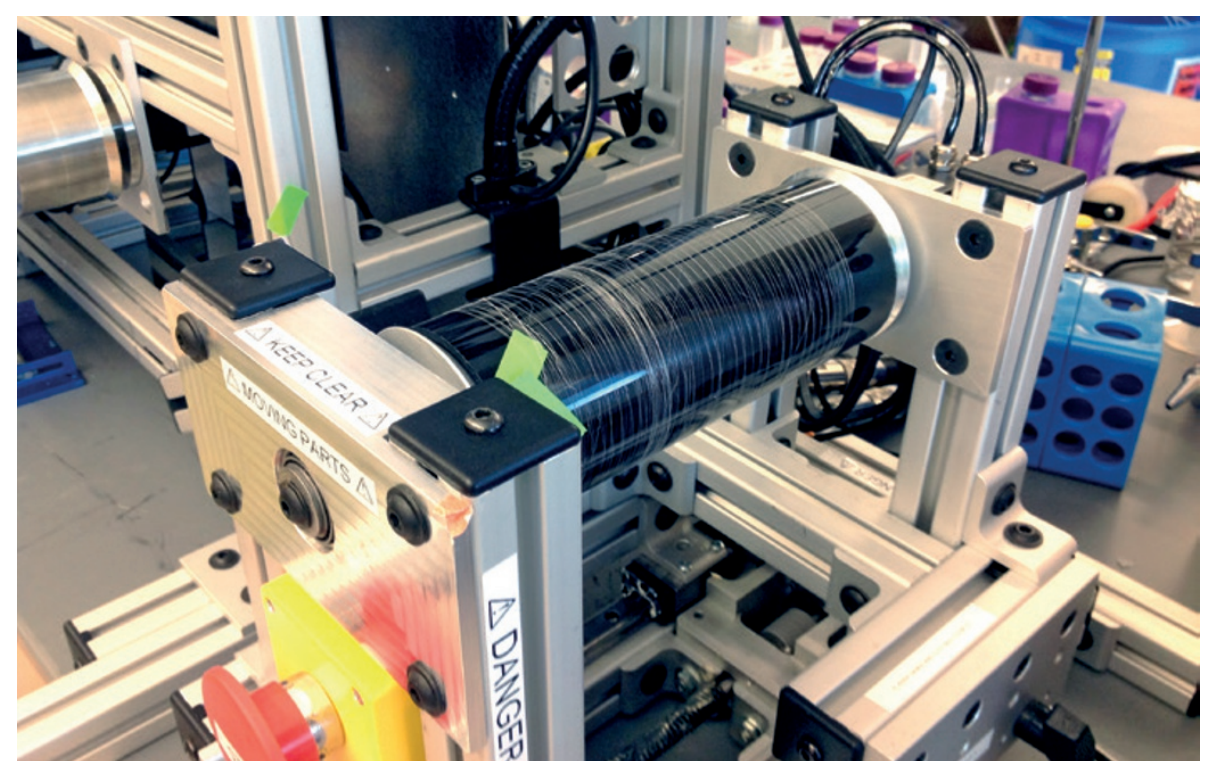

Artificial silk is spun from genetically engineered spider-silk proteins at Utah State University in Logan.

that is also known as the golden silk orbweaver. Since then, Lewis and others have succeeded in expressing this protein in many production systems, including the bacterium Escherichia coli, alfalfa and goats, which express the molecule in their milk. Lewis has started a company called Araknitek to develop and license these proteins.

There are other companies developing spider-silk proteins. AMSilk, which is based near Munich, Germany, manufactures synthetic spider-silk proteins for use in cosmetics and shampoos; Spiber Technologies, based in Stockholm, is selling meshes and films for growing cell cultures consisting of laboratory-made spider silk.

Another almost identically named company - Spiber Inc. in Tsuruoka, Japan - has spun off from Keio University. Many commercially minded companies are developing films or fibres of artificial spider silk for use in medical devices and textiles.

But no one has figured out how to produce spools of silk as tough as the original. "Our best fibres have about half to two-thirds the tensile strength of spider silk," says Lewis. One problem is that engineered spider-silk protein molecules are not as large as the originals. A general rule of fibre-spinning, whether the materials are proteins or plastics, is that the larger the molecules, the stronger the fibre. But this trend bumps up against an axiom of bioengineering: the bigger the protein, the more difficult it is to produce in a genetically modified organism. Natural dragline proteins have molecular masses of 250 to 500 kilodaltons; genetically modified organisms made in the laboratory produce silk proteins of only about 60 to 80 kilodaltons. Lewis says that he is continuing to work on producing proteins two to three times that size, much closer to natural spider-silk proteins, in genetically modified organisms. "The tensile strength is going to improve," he says.

Even with the right proteins, humans are at a disadvantage in putting them together because we still do not fully understand how spiders do it. Artificial silk fibre-spinning techniques are based on the methods used to make polymer fibres such as Kevlar. These techniques work well for the artificial polymers they were designed for but not as well for proteins. The proteins are pushed out of the tip of a fine needle, washed in baths of water or alcohol, then stretched and dried - a process very different from the method spiders use. Scientists know that spiders pull silks out of dedicated glands, some of which contain more than one kind of protein. The silk emerges from the gland as a highly concentrated liquid solution of fibres that solidifies as it is pulled out. This process has not yet been mimicked, and researchers are only beginning to understand the details of anatomy and physiology that make silk possible.

Anna Rising, a biochemist at the Swedish University of Agricultural Sciences in Uppsala, is turning to spiders for clues on fibre spinning. To learn more about how to make tough silk fibres, she and a student performed an intricate study ${ }^{3}$ that they hope will lead to insights that can be applied to industrial silk-making. They placed microelectrodes along a living spider's silk glands, from the bag-like reservoir down the narrowing tube to its opening. These sensors revealed that "there's quite an impressive $\mathrm{pH}$ gradient and an increase in $\mathrm{CO}_{2}$ " along the length of the gland, she says. Rising is now working on replicating these gradients, which she says probably help to control the crystallization of the silk proteins as they are spun. 
Rising has also found that, while inside the gland, the head of one silk protein attaches to the tail of the next in such a way that groups of them line up in a row ${ }^{4}$. As the proteins are assembled inside the gland, they pre-align. The better aligned the proteins are in a fibre, the stronger it is. This might also be mimicked in synthetic fibres. Swedish company Spiber is working to commercialize Rising's ideas, producing recombinant silk proteins in E. coli with the goal of making replacement tendons and ligaments for clinical use.

Hayashi hopes more insights will come from watching the silk gland develop, as young spiders mature into adults. She is studying Latrodectus hesperus, commonly called the black widow spider. This species has seven types of silk glands, each of which it draws on to make a unique silk fibre.

Although many researchers have been inspired by the mechanical toughness of spider draglines, others see broader potential in spider silks. Todd Blackledge, an entomologist at the University of Akron in Ohio, was the first to characterize the silk of Darwin's bark spider. He says that focusing on just one spider or one kind of silk would be like working only with inbred white laboratory rats. $\mathrm{He}$ is looking at silks made by other spiders, and is working with Akron materials scientist Ali Dhinojwala to study what makes some spiderwebs sticky. The pair have found that, for many silks, stickiness comes not only from chemistry but also from architecture.

One common arachnid adhesive technique is to make a patch of tightly packed, parallel silk threads to secure the dragline at one end. Spiders do this so that they may safely dangle. The adhesive patch is made of parallel threads of a coil-shaped silk protein called pyriform silk; fibres made from this protein are highly elastic, like a rubber band. The pyriform silk is coated with a sticky protein solution. Removal of this kind of dense adhesive patch requires pulling hundreds of elastic silk strands past their breaking point. Dhinojwala, a polymer chemist, mimicked this in a polymer adhesive patch. He showed that a patch of polymer threads with the right elasticity is sticky like the spider patches and hopes to develop artificial patches for use in surgical repair of tendons.

\section{BEYOND SPIDERS}

Whereas some researchers have been untangling the challenges of spider silks, others have been taking advantage of the properties of silks made by an animal that is easier to work with. The silkworm, Bombyx mori, has long been domesticated for the production of lustrous textiles.

Silkworm fibre is not as tough as spiders' silks and is in many ways simpler. The insects do only one thing with their silks: build cocoons for shelter during metamorphosis. And their silk has just one structural

\section{THE TOUGHEST THREAD}

Seemingly delicate spiderwebs are constructed from incredibly robust silk fibres that surpass artficial materials in toughness (the amount of energy they can absorb before breaking).

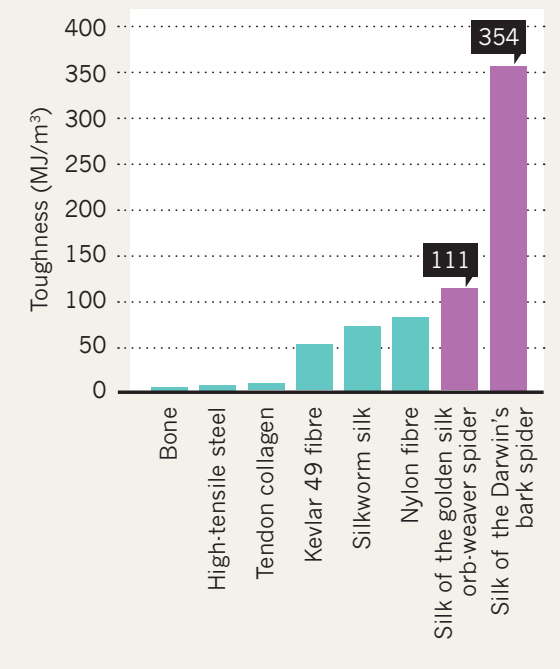

component, called fibroin. Kaplan sees the potential of fibroin - and it is not necessarily in the form of fibres.

Silkworm fibroin is readily available and easy to work with, says Fiorenzo Omenetto, a Tufts biomedical engineer with expertise in photonic materials. To compete with commodity materials such as plastics and silicon for applications in electronics and medical devices, scale is key. Omenetto orders huge boxes of silkworm cocoons, then processes them according to a recipe originally developed by Kaplan. The

"Sill is the best stuff on Earth. There's nothing like it." cocoons are cut open and the pupae removed. Then the cocoons are put into a hot bath to wash the silk fibres clean of the gluey glycoprotein sericin, before being processed to dissolve and purify the fibroin protein.

The thick, water-based solution that results can be mixed with drugs or other biomolecules or nanoparticles, then moulded into films or other structures. By controlling the conditions during the drying step, researchers can direct the crystallization of fibroin into forms that will dissolve on a desired timescale inside the body.

Kaplan and Omenetto have made silk films to house biodegradable electronic devices that monitor heart function and then dissolve $^{5}$. They have also made drug-releasing implants, artificial corneas, silk orthopaedic hardware strong enough to screw into bone and then degrade over time, and squishy scaffolds for growing neurons from the brain's cerebral cortex.

Medical applications are paving the way for technological uses of silk, says Kaplan. Silk thread is already used extensively in medical settings, where it is the most commonly used suture material. And some silk biomedical devices made by Kaplan and other researchers are on the market or in clinical trials for softtissue reconstructive surgery. Furthermore, Orthox in Oxfordshire, UK, is testing silk devices for knee repair. Many of the researchers working on producing spider silk would like to use it in similar applications, such as repairing ligaments, where its extra toughness would be an additional bonus.

Tara Sutherland, a bioengineer at the Commonwealth Scientific and Industrial Research Organization in Canberra, Australia, is looking to silks that are less well known than those from spiders and silkworms. Sutherland's analysis ${ }^{6}$ has uncovered 23 groups of independently evolved types of silk in 100,000 different insect species. In common with Kaplan, Sutherland has medical applications in mind. She zeroed in on one insect from thousands of options: the honeybee. Honeybees use silk to provide thermal insulation and structural support for their hives.

Sutherland approached the problem of picking the right silk from a bioinformatics point of view, not an evolutionary one. She wanted to make the most of what biopolymers offer. Unlike plastics and other materials, proteins can sense and respond to the environment. Sutherland wants to make tissue-engineering scaffolds that release cellattracting signals on cue, or silk bandages that release antibiotics when they sense an infection. She has funding from the clothing firm Nike and NASA, among others.

Seeking a silk that would be easy to modify to add smart features, her analysis pointed to the type produced by honeybees. These proteins are smaller than those found in spider silks and so are easier to genetically engineer. Sutherland's analysis showed that huge stretches of the genetic sequence could be altered without interfering with the proteins' toughness and biodegradability. That gives her more room to engineer in new functions, such as attracting cells to help rebuild tissue. Sutherland is now developing bioactive bandages and other medical devices that use these silks.

For Sutherland and others, the fibres produced by silk makers in the wild are endlessly fascinating and full of design ideas. "Silk is the best stuff on Earth," says Kaplan. "There’s nothing like it."

Katherine Bourzac is a freelance science writer in San Francisco, California.

1. Gregorič, M., Agnarsson, l., Blackledge, T. A. \& Kuntner, M. PLoS ONE 6, e26847 (2011).

2. Xu, M. \& Lewis, R. V. Proc. Natl Acad. Sci. USA 87, 7120-7124 (1990).

3. Andersson, M. et al. PLoS Biol. 12, e1001921 (2014).

4. Kronqvist, N. et al. Nature Commun. 5, 3254 (2014).

5. Tao, H. et al. Proc. Natl Acad. Sci. USA 111, 17385-17389 (2014)

6. Sutherland, T. D., Young, J. H., Weisman, S., Hayashi, C. Y. \& Merritt, D. J. Annu. Rev. Entomol. 55, 171-188 (2010). 